\title{
Plasmonic behaviour of sputtered Au nanoisland arrays
}

\author{
V. Tvarožek ${ }^{a}$, O. Szabóa, ${ }^{a}$, I. Novotný $^{a}$, S. Kováčováa ${ }^{a}$, J. Škriniarováa $^{a}$, P. Šutta ${ }^{b}$ \\ a Institute of Electronics and Photonics, FEI STU, Ilkovičova 3, SK-812 19 Bratislava, Slovakia \\ ${ }^{\mathrm{b}}$ New Technologies-Research Centre, University of West Bohemia, Univerzitní 8, 30614 Plzeň, Czech Republic
}

\section{A R T I C L E I N F O}

\section{Article history:}

Received 25 January 2016

Received in revised form 28 April 2016

Accepted 29 April 2016

Available online $\mathrm{xxx}$

\section{Keywords:}

Sequential sputtering

Au nanoisland arrays

Localised surface plasmon resonance

Universal thickness scale rule of plasmon

resonance

\begin{abstract}
A B S T R A C T
The specificity of the formation of Au sputtered nanoisland arrays (NIA) on a glass substrate or on a ZnO thin film doped by Ga is demonstrated. Statistical analysis of morphology images (SEM, AFM) exhibited the Log-normal distribution of the size (area) of nanoislands-their modus $A_{M}$ varied from 8 to $328 \mathrm{~nm}^{2}$ depending on the sputtering power density, which determined the nominal thicknesses in the range of 2-8 $\mathrm{nm}$. Preferential polycrystalline texture (111) of Au NIA increased with the power density and after annealing. Transverse localised surface plasmonic resonance (LSPR; evaluated by transmission UV-vis spectroscopy) showed the red shift of the extinction peaks $(\Delta \mathrm{l} \leq 100 \mathrm{~nm})$ with an increase of the nominal thickness, and the blue shift ( $\Delta \lambda \leq-65 \mathrm{~nm}$ ) after annealing of Au NIA. The plasmonic behaviour of Au NIA was described by modification of a size-scaling universal model using the nominal thin film thickness as a technological scaling parameter. Sputtering of a Ti intermediate adhesive ultrathin film between the glass substrate and gold improves the adhesion of Au nanoislands as well as supporting the formation of more defined Au NIA structures of smaller dimensions.
\end{abstract}

(C) 2016 Elsevier B.V. All rights reserved.

\section{Introduction}

Noble metal nanostructures have been widely investigated in label-free biological sensor applications [1,2] due to localised surface plasmon resonance (LSPR), which is a coherent oscillation of the free electron gas in metal nanoparticles excited by electromagnetic radiation, particularly in the optical range. Trends in the development of plasmonic technologies include efforts to produce Au/Ag nanoparticles of controllable shape, size and porosity with the aim of improving LSPR tunability and of simplifying the technological procedure [3]. Innovative physical technologies have been developed recently, as an alternative to traditional chemical reduction methods for obtaining metal ( $\mathrm{Au}, \mathrm{Ag}$ ) nanoparticles: laser ablation/pulsed deposition, electron-beam/nanosphere lithography [4]; thermally induced dealloying and dewetting on pre-patterned substrates by nanoimprint lithography [5], electronbeam [6] and evaporation [7] depositions of Au island films, and sputtering of nanostructured/porous Au films and nanoisland arrays [8-10]. The application of LSPR sensors requires a solid transparent support, e.g., a glass substrate or transparent conductive oxide (TCO) films. ZnO doped by Ga (GZO) belongs to TCO films

\footnotetext{
* Corresponding author.

E-mail address: ondrej.szabo@stuba.sk (P. Šutta).
}

that have attracted attention due to their nontoxic nature, costeffectiveness and easy fabrication [11].

Our aim was to simplify the technological procedures of the formation of Au plasmonic nanostructures (island-like shape) directly by sputtering without masking and lithography. The structural, morphological and optical properties of sputtered Au nanoisland arrays (on Corning glass or GZO/Corning glass substrates) were studied and compared to the universal size-scaling plasmon coupling model [12,13]. Moreover, the description of the plasmonic behaviour of sputtered Au NIA is presented by using thin film technological parameters, such as the RF power density and the nominal thickness.

\section{Experimental techniques}

We used the deposition concept of sequential (cyclic) sputtering [14]: Fig. 1. In this dynamic mode of deposition, the substrate holder rotated under the target. The RF diode sputtering system Perkin/Elmer 2400/8L was used for deposition of GZO films and Au NIA on Corning glass substrates. The nominal thicknesses of Au island films (2-8 nm)-sputtered from an Au target with diameter $203.2 \mathrm{~mm}$ at RF power 75-300 W-were determined by a Dektak profilometer. Post-deposition annealing was carried out in the sputtering chamber immediately after deposition $\left(300^{\circ} \mathrm{C} / 2\right.$ h) and in an external furnace (air: $500^{\circ} \mathrm{C} / 5 \mathrm{~h}$ ). The morphology of the nanostructures was analysed by scanning electron 


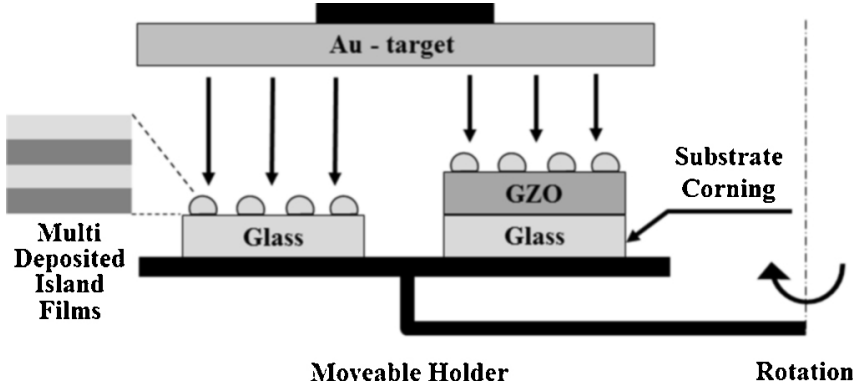

Fig. 1. Schematic layout of sequential sputtering.

microscopy (SEM) JEOL 7500F and by atomic force microscopy (AFM) PARK XE 10. Their crystalline structure was investigated by X-ray diffraction (XRD) using the X-ray diffractometer X'Pert Pro with a Bragg-Brentano goniometer and Copper $K \alpha$ radiation source $(\lambda=0.154 \mathrm{~nm})$. UV-vis transmission spectra was measured using Ocean Optics 4000 UV-vis Spectrometer in the spectral range $300-900 \mathrm{~nm}$.

\section{Results and discussion}

We have to point out the specificity of the formation of nanostructures by exploiting early stages of thin-film growth. Sputtered non-continuous island Au films were grown according to the Volmer-Weber model [15]: for the formation of nuclei, clusters

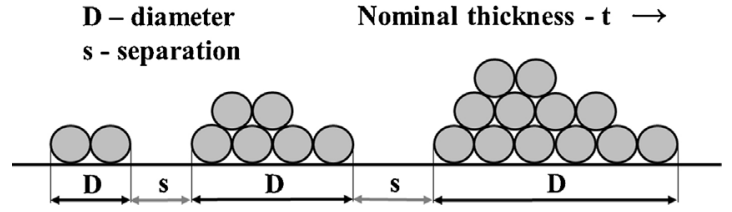

Fig. 2. Schematic model of the early growth stage of sputtered Au island films.

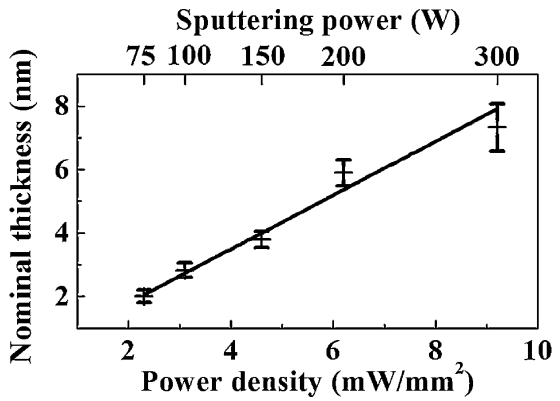

Fig. 3. Nominal thickness of Au nanoisland films vs. RF sputtering power (RF power density).

and islands and their coalescence, see Fig. 2. The nominal thickness measured by the mechanical Talystep method gives the value that characterises only the "envelope" of the NIA, which implicitly contains data of the amount of deposited material, its distribu-

Au on Corning glass
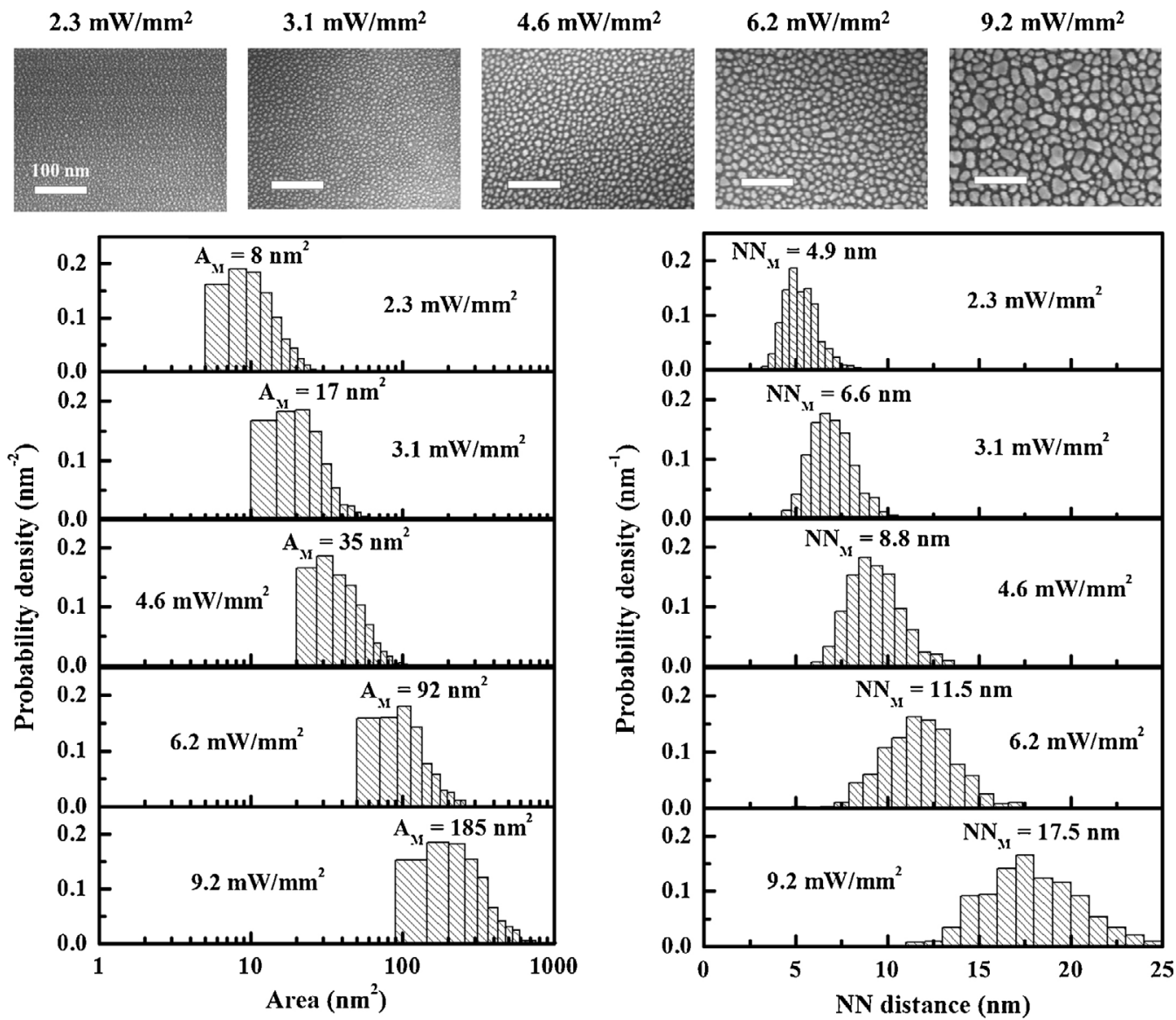

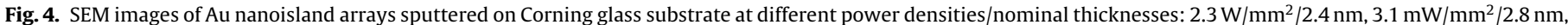

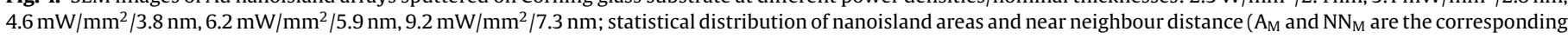
moduses). 


\section{Au on GZO thin film}

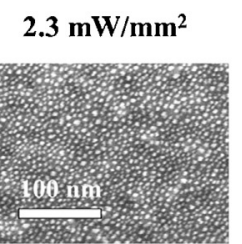

\section{$4.6 \mathrm{~mW} / \mathrm{mm}^{2}$}
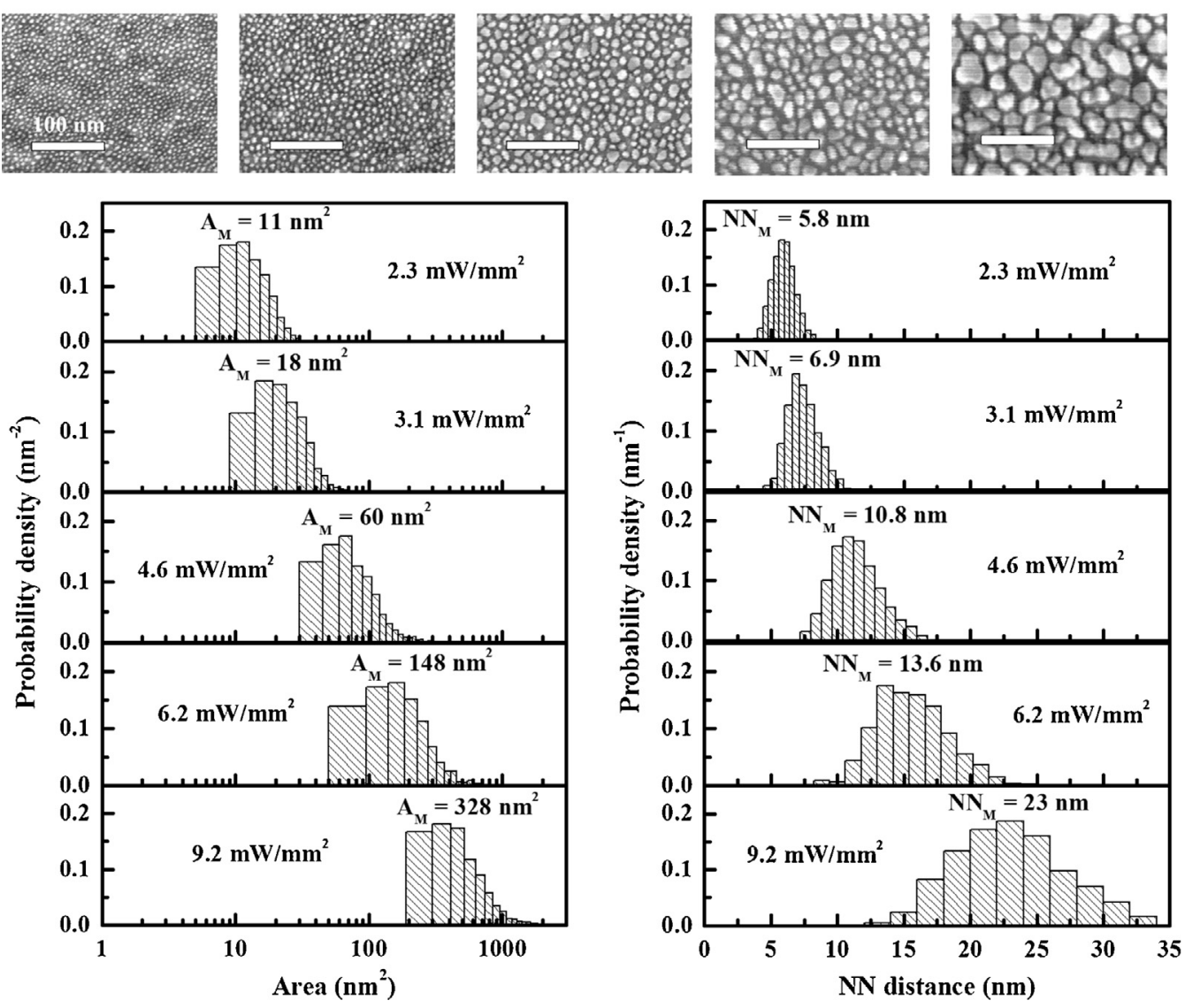

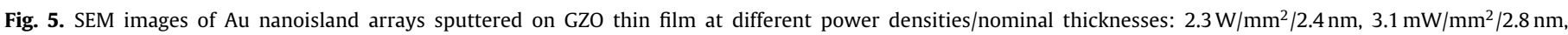

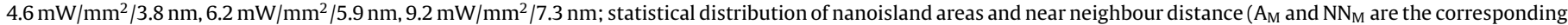
moduses).

tion, structure and size, including the height of the nanoislands. Their nominal thicknesses were near-linearly proportional to the sputtering RF power (RF power densities): Fig. 3.

SEM and the open source image processing program Image [16] were used to evaluate the surface morphologies. Statistical analysis of SEM images of Au surface morphology (Figs. 4 and 5) exhibited the Log-normal distribution of the area of NIA and the Gaussian distribution of the near neighbour distance of NIA.

The centre of the Log-normal distribution of the nanoisland areas is modus $A_{M}$ and the centre of the Gaussian distribution of the near neighbour distance between nanoislands is modus $N N_{M}$ (i.e., the value that appears most often in the set of data). An increase of the nominal thickness caused an evident increase of the nanoisland areas (diameters) (Figs. 4 and 5), but their separations were extended only a little. This varied either from 8 to $185 \mathrm{~nm}^{2}$ (Corning glass substrate, Fig. 4) or from 11 to $328 \mathrm{~nm}^{2}$ (GZO thin film/Corning glass substrate, Fig. 5) depending on the RF sputtering power density. In both cases, the nanoisland NN distance exhibited a Gaussian distribution. We used very rough approximations to determine the nanoisland dimension $D$ (assuming a circular shape of the NIA area $\left.\left(D=2 .\left(\sqrt{ } A_{M} / \pi\right)\right)\right)$ and inter-island separations $s$ from the statistical evaluation SEM images $\left(s=N N_{M}-D\right)$. The separation and diameter of Au NIA depending on RF power density are shown in Fig. 6 . The diameter of Au NIA was changed significantly from $4 \mathrm{~nm}$ to $20 \mathrm{~nm}$, while their separation changed a little from $2 \mathrm{~nm}$ to $4 \mathrm{~nm}$, with an increase of the sputtering power density. The comparison of NIA sizes led to the conclusion that the GZO thin film enhanced the

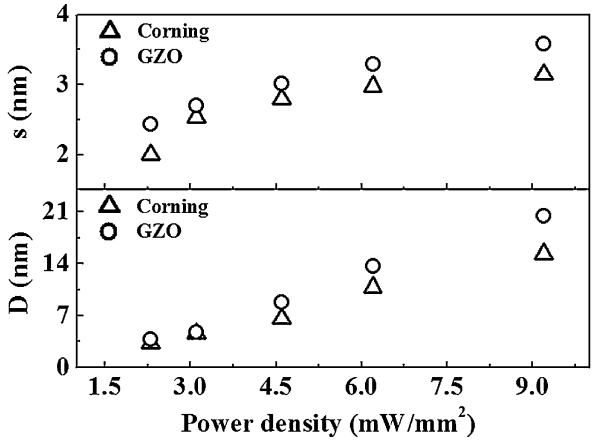

Fig. 6. Separation and diameter of Au NIA vs. RF power density.

growth of greater nanoislands, compared to the nanoislands grown on the Corning glass substrate.

The XRD analysis indicated the polycrystalline structure of $\mathrm{Au}$ NIA preferentially oriented in the [111] direction, Fig. 7. The texture of Au NIA increased with the power density.

The transverse localised surface plasmon resonance (LSPR) and the scattering were evaluated by transmission UV-vis spectroscopy, Fig. 8.

The minimum in the optical transmittance curves represents the LSPR absorption corresponding to the extinction peak in the extinction spectra (extinction is defined as a decadic logarithm of transmission). This phenomenon is the consequence of collec- 

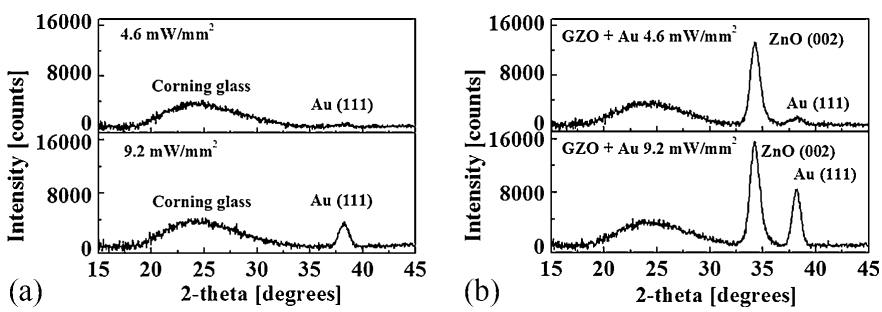

Fig. 7. Examples of XRD lines of Au NIA sputtered on: (a) Corning glass substrate, (b) GZO/Corning glass substrate-at RF power densities of $4.6 \mathrm{~mW} / \mathrm{mm}^{2}$ and $9.2 \mathrm{~mW} / \mathrm{mm}^{2}$.

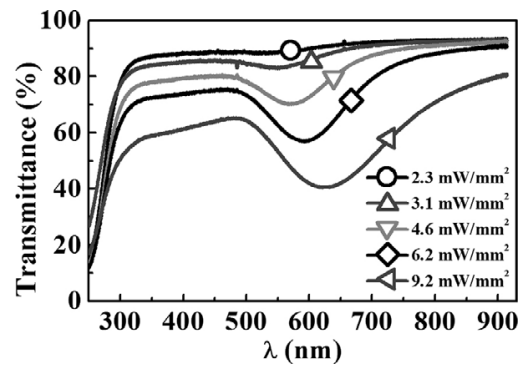

Fig. 8. Example of the transmittance spectrum of Au NIA on the Corning glass substrate (RF power density as a parameter).
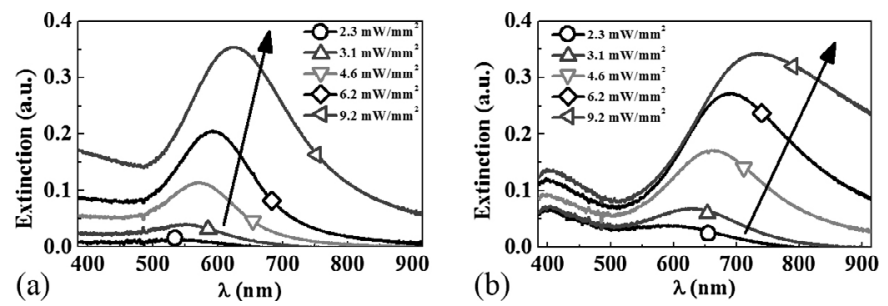

Fig. 9. Influence of RF sputter density on extinction spectra of Au NIA sputtered on: (a) Corning glass substrate and (b) GZO thin film/Corning glass substrate.

tive oscillation of electrons in Au NIA due to optical radiation. The coupled plasmon resonance wavelength $\lambda$ depends on nanostructure size, shape, orientation and local dielectric environment in the whole range of visible to NIR spectral regions [12,17].

The LSPR showed the red shift of extinction peaks $(\Delta \lambda \leq 100 \mathrm{~nm})$ with an increase of sputtering power density (Fig. 9a, b), meaning an increase of the size of Au nanoislands particularly. Our experimental results lead to the conclusion that our optical measurements showed the limitation regarding the Au NIA sputtered at very low power densities.

Our results are comparable to the universal size-scaling model of LSPR shift [17], where the fractional shift $\Delta \lambda / \lambda_{0}$ of the plasmon resonance wavelength varies with the ratio of interparticle separation $s$ and particle dimension $D$. The $s / D$ ratio characterises the inherent scaling of two competitive forces: plasmon dipolar strength (polarizability) and interparticle Coulombic restoring strengths. Based on these phenomena, the universal size-scaling plasmon rule equation was derived:

$\frac{\Delta \lambda}{\lambda_{0}}=\operatorname{kexp}\left(-\frac{1}{\tau} \frac{s}{D}\right)$

when $\Delta \lambda=\lambda-\lambda_{0}$ is the wavelength shift, $\lambda_{0}$ is the single-particle resonance wavelength for a very large $s / D$ ratio, $\tau \approx 0.2$ (the most related value) is the exponential decay constant.

The change of the plasmon resonance wavelength with the NIA $A_{M}$ formed on Corning glass substrate and GZO film is shown in Fig. 10. We defined the reference resonant wavelength $\lambda_{0}$ as the minimum detectable value in our measurements.

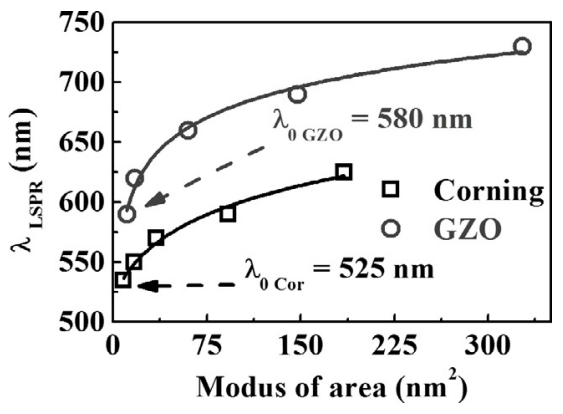

Fig. 10. Change of the plasmon resonance wavelength with the NIA $A_{M}$.

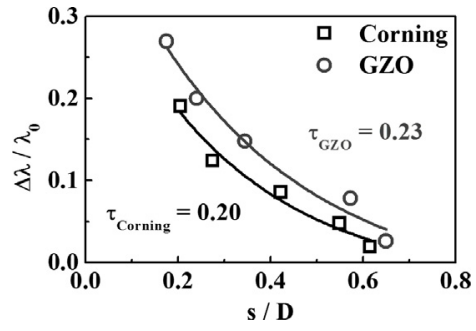

Fig. 11. Exponential dependence of the universal size-scaling rule (Equation 1) for both NIA formed on the Corning glass and GZO film substrates.

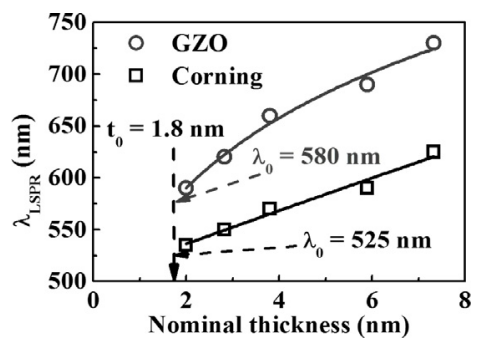

Fig. 12. LSPR wavelength dependence on the nominal thicknesses of Au NIA sputtered on Corning glass/GZO thin film substrates.

In spite of previously mentioned arguments, the fractional shift $\Delta \lambda / \lambda_{0}$ of the plasmon resonance wavelength followed the exponential dependence of the universal size-scaling rule for NIA formed both on the Corning glass and GZO film substrates, Fig. 11. The decay constant $\tau$ was close to 0.2 .

We tried to evaluate the LSPR response of Au NIA from the thin film technology point of view. In our semi-empirical approach, the nominal thickness $t$ was used for scaling. It implicitly characterises both the amount of deposited material and the distribution of NIA (area/separation). Since the nominal thickness was linearly proportional to RF power density (Fig. 3), the curve of the LSPR wavelength versus the nominal thickness (Fig. 12) had the same tendency as the dependence of LSPR wavelength on the island area $A_{M}$ (i.e., RF power density, Fig. 10).

Therefore, we normalised nominal thicknesses to the reference thickness $t_{0}$ which corresponds to the reference resonant wavelength $\lambda_{0}$ as the minimum detectable value in our measurements. We found that the $s / D$ ratio derived from the statistical evaluation of the SEM surface images was linearly proportional to the reciprocal thickness ratio $t_{0} / t$ (Fig. 13). This fact enabled us to replace formally parameter $s / D$ in Equation (1) by the ratio $t_{0} / t$. This is only a phenomenological parameter that has no direct physical meaning as regards plasmon coupling strength. 


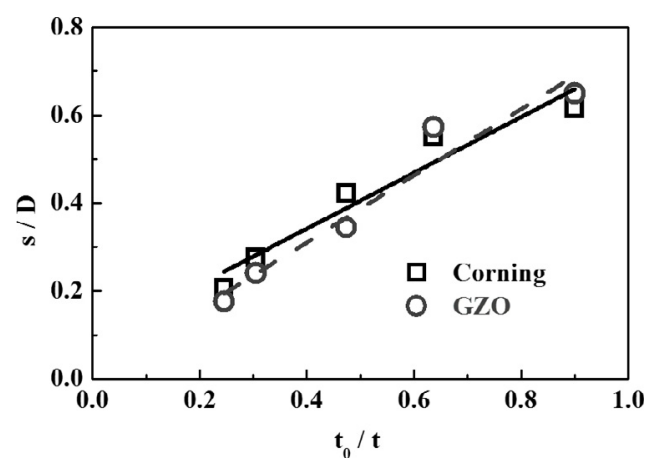

Fig. 13. Relation between the scale factor $s / D$ and the reciprocal thickness $t_{0} / t$ of $A u$ NIA.

Then, the variation of the plasmon resonance wavelength fractional shift $\Delta \lambda / \lambda_{0}$ with the reciprocal relative thickness change $t_{0} / t$ can be described by the exponential dependence

$\frac{\Delta \lambda}{\lambda_{0}}=\operatorname{kexp}\left(-\frac{1}{\tau_{t}} \frac{t_{0}}{t}\right)$

where the constant $\tau_{t}$ mathematically describes the decay of the plasmon resonance wavelength fractional shift $\Delta \lambda / \lambda_{0}$ with nominal thickness, Fig. 14.

The comparison of NIA formed on the Corning glass and GZO film/Corning glass substrates - besides the influence of another morphology - shows also the refractometric effect of the substrate on the LSPR shift (Figs. 9-12). The refractive indices of Corning glass $(\approx 1.5)$ and of GZO $(\approx 2.0)$ are different, which tends to shift the LSPR to longer wavelengths (including the reference single-particle resonance wavelength $\lambda_{0}$ ). Such a difference breaks the symmetry of the electromagnetic field distribution around the nanoisland (it is concentrated in the higher refractive index region), as confirmed experimentally and by simulations [18].

Thermal treatment of nanostructures after deposition is a wellknown and important technological step to obtain $\mathrm{Au}$ or $\mathrm{Au} / \mathrm{Ag}$ nanoparticles of a desired size, shape and structure $[3,6-8,10]$. External annealing $\left(500^{\circ} \mathrm{C} / 5 \mathrm{~h}\right.$ in air) of our thicker Au NIA (thick-
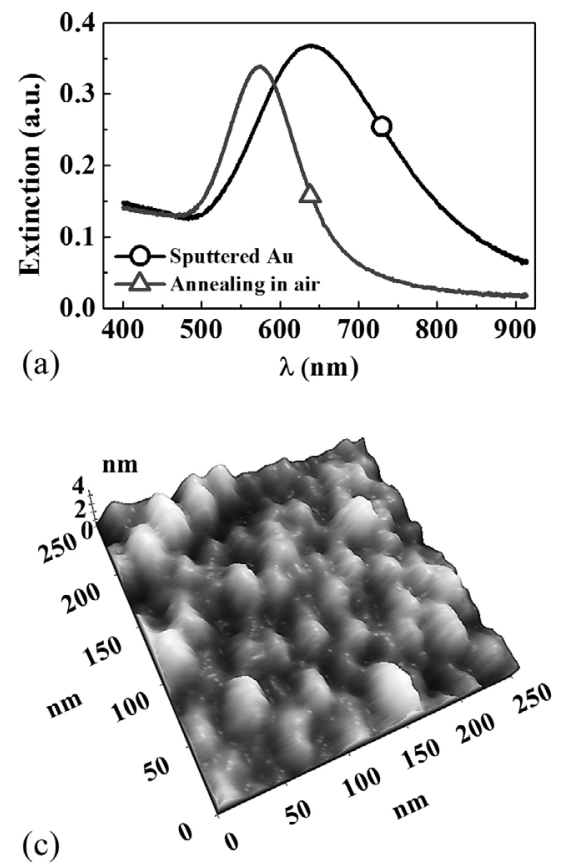

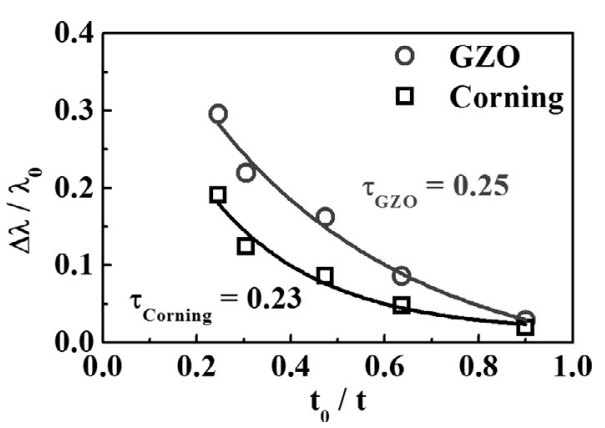

Fig. 14. Exponential decay of plasmon resonance wavelength fractional shift for both NIA formed on the Corning glass and GZO film/Corning glass substrates.

ness of $7.3 \mathrm{~nm}$ ) had a pronounced effect on the morphology and LSPR. Extinction spectra exhibited the LSPR blue shift, $\Delta \lambda \leq-65 \mathrm{~nm}$, after annealing of Au NIA (Fig. 15a), due to the coalescence of small and closely spaced nanoislands. An increase of the granularity of surface morphology and coagulation of Au particles into larger aggregates was also confirmed by AFM analyses (Fig. 15c, d).

The energy delivery during the heat treatment had mainly two effects on the films analysed: (i) growth of the gold nanoparticles due to the coalescence (clearly observed by AFM and SEM) and (ii) narrower width of X-ray diffraction line profiles, indicating higher dimensions of coherently diffracting domains (crystallites) and lower values of micro-strains. The heat treatment annealed some local structure imperfections within the crystallites volume, which have their activation energies corresponding to the annealing temperatures used (Fig. 15b).

The structural and optical properties of the RF diode sputtered Au NIA were comparable to the results obtained by evaporation of ultrathin $\mathrm{Au}$ island films [6,7], RF diode sputtering of Au nanostructures [8], and cyclic sputtering and anneal of isolated/well-dispersed Au nanoisland arrays [10].

We sputtered $\mathrm{Ti}$ as an intermediate non-continuous film (approximately $0.6 \mathrm{~nm}$ in nominal thickness) between the Corning glass substrate and gold, with the aim of improving the adhesion
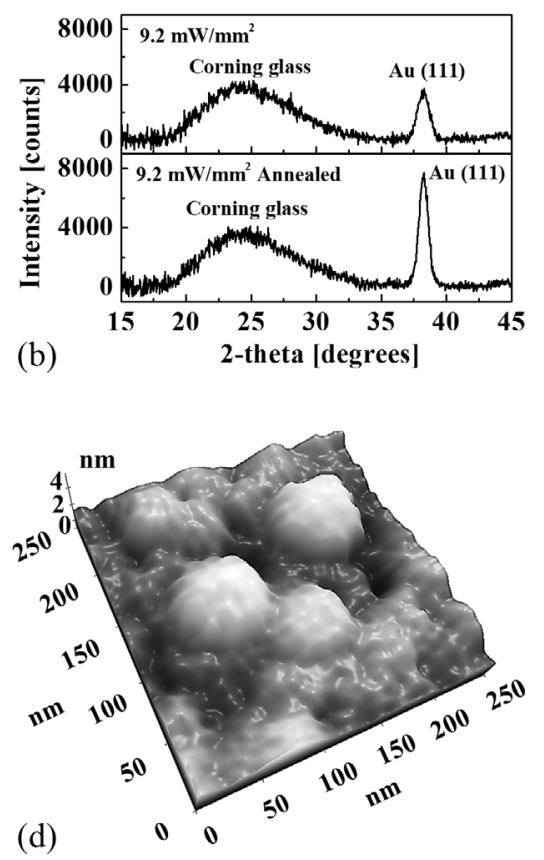

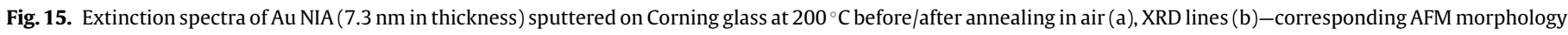
before (c) and after (d) annealing of Au NIA. 


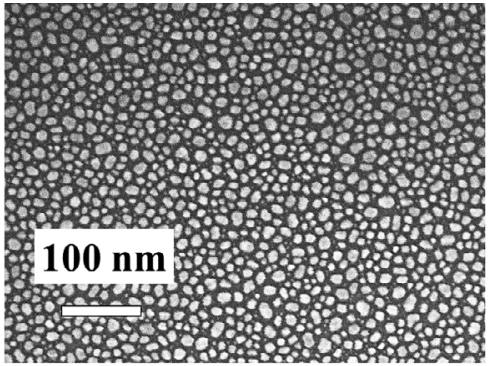

(a)
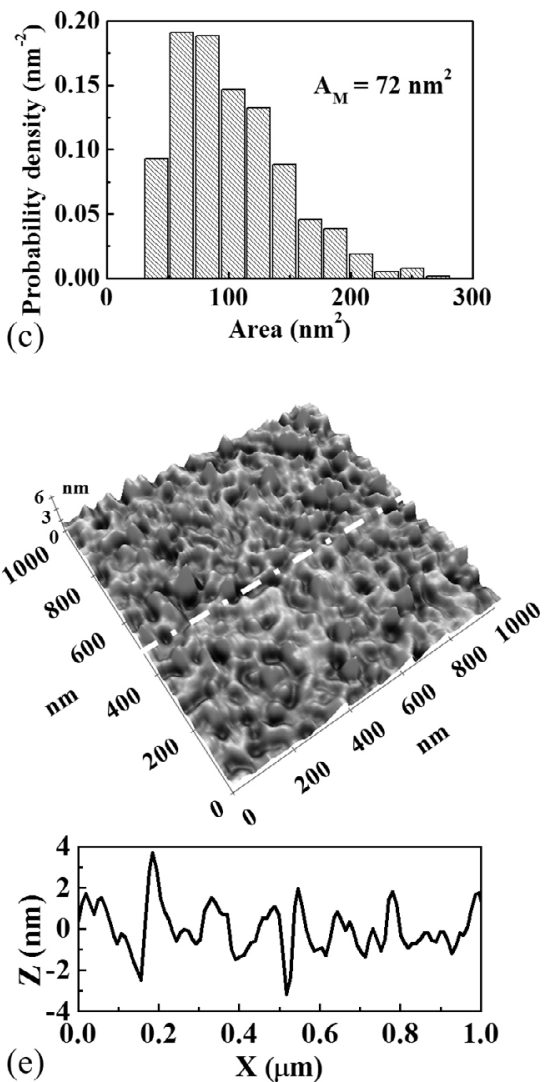

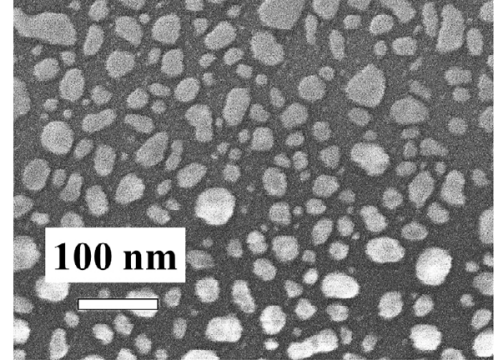

(b)

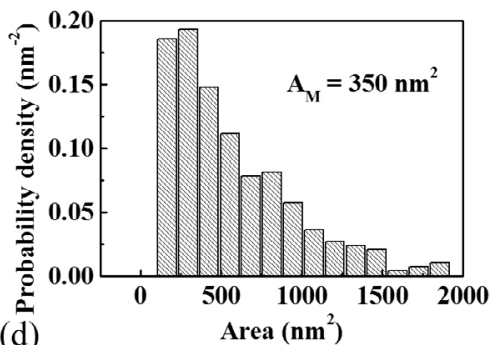

(d)
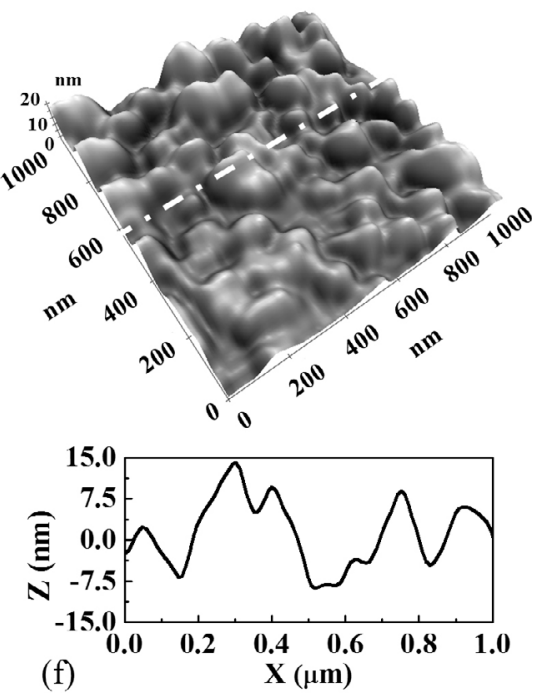

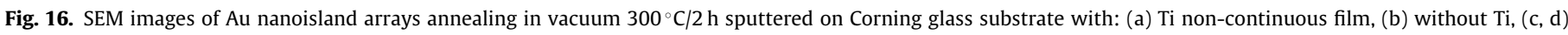
Statistical analysis of SEM morphology and (e, f) AFM images of Au nanoislands.

of $\mathrm{Au}$ NIA. Au NIA of the same nominal thickness of $6 \mathrm{~nm}$ were sputtered on Corning glass (with or without $\mathrm{Ti}$ ) by a power density $2.3 \mathrm{~mW} / \mathrm{mm}^{2}$ at a substrate temperature $200^{\circ} \mathrm{C}$; after deposition they were immediately annealed in a vacuum at $300^{\circ} \mathrm{C} / 2 \mathrm{~h}$ (Fig. 16). The Ti adhesive non-continuous film created very small "seeds" for catching deposited Au atoms. After application of the Ti adhesive layer, Au NIA had more uniform distribution; they were smaller with more defined shape (coming near to circular islands). The formation of more defined and well-dispersed Au/Ti NIA structures of smaller areas $\left(A_{M}=72 \mathrm{~nm}^{2}, s=2.9 \mathrm{~nm}\right.$. $\left.s / D=0.31\right)$ took place (Fig. 16a, c), compared to the single Au NIA $\left(A_{M}=350 \mathrm{~nm}^{2}\right.$, $s=5.9 \mathrm{~nm}, \mathrm{~s} / \mathrm{D}=0.28$ ), Fig. $16 \mathrm{~b}, \mathrm{~d}$. The positive effect of Ti upon the formation of stable Au NIA was also confirmed by AFM analyses (Fig. 16e, f). As a consequence, the scaling ratio $s / D$ increased in the case of Au/Ti NIA, and a blue shift of LSPR wavelengths was observed, Fig. 17. The adhesive non-continuous film of Ti caused only a small decrease of the intensity of LSPR extinction spectra compared to their significant broadening and intensity reduction due to $\mathrm{Ti}$ or $\mathrm{Cr}$ adhesion layers with thicknesses of $\geq 1 \mathrm{~nm}$ [18]. This fact is important for application of the Au/Ti NIA in LSPR biosensing.

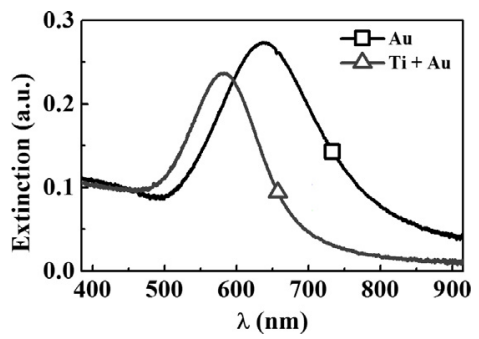

Fig. 17. Extinction spectra of Au NIA annealing in vacuum $300^{\circ} \mathrm{C} / 2 \mathrm{~h}$ sputtered on Corning glass substrate at $200^{\circ} \mathrm{C}$ with and without Ti.

They were also more stable and relatively insensitive to temperature treatment.

We found that sputtering at an elevated substrate temperature $\left(200^{\circ} \mathrm{C}\right)$ or thermal treatment $\left(300^{\circ} \mathrm{C} / 2 \mathrm{~h}\right.$ in vacuum) in the sputtering chamber immediately after deposition is sufficient to obtain stable isolated Au NIA. Moreover, Au NIA externally annealed (Fig. 15) had the same maximum peaks in the extinc- 
tion spectra as sputtered Au NIA with the Ti intermediate adhesion non-continuous film without annealing in air, Fig. 17. This indicates the positive effect of Ti on the formation of stable Au NIA. Exclusion of external annealing was possible in the case of small sizes of Au nanoislands $(<10 \mathrm{~nm}$ ) on Ti, which were completely prepared in the sputtering chamber (i.e. internal heating of substrate). This is not valid for larger nanoparticles and other technologies $[3,8,10]$.

\section{Conclusions}

The specificity of the formation of Au sputtered nanoisland arrays was demonstrated: (i) the size and shape of Au nanoislands, as well as their separations, were particularly controlled by the sputtering power density and Au nominal thickness; (ii) the size of gold nanoisland exhibited the Log-normal distribution, and their separation showed the Gaussian distribution; these parameters were interactively dependent. The structural, morphological and optical properties of sputtered Au nanoislands were compared to the universal size-scaling plasmon coupling model, and were consistent with today's empirical and theoretical knowledge.

The plasmonic behaviour of Au NIA was described by the modification of the size-scaling universal model using thin-film technological parameters, particularly the nominal thin-film thickness.

Sputtering of a Ti intermediate adhesive non-continuous film between Corning glass substrate and gold improves the adhesion of Au NIA, as well as supporting the formation of more defined Au NIA structures of smaller dimensions. They are more stable and relatively insensitive to temperature treatment.

The formation of sputtered Au plasmonic nanostructures on transparent substrates, including conductive $\mathrm{ZnO}$ thin films, paves the way for their exploitation in biosensors and photovoltaic solar cells.

\section{Acknowledgements}

This work was supported by SRD Project No. APVV-036512, SK VEGA Project 1/0739/16, SDR APVV 0395/12 and was partially developed within the CENTEM project Reg. No. CZ.1.05/2.1.00/03.0088 and CENTEM PLUS (LO1402) project.

\section{References}

[1] J.N. Anker, W.P. Hall, O. Lyandres, N.C. Shah, J. Zhao, R.P. van Duyne, Biosensing with plasmonic nanosensors, Nat. Mater. 7 (2008) 442-452.

[2] L. DeSioa, T. Placido, R. Comparellid, M.L. Currid, M. Striccolid, N. Tabiryana, T.J. Bunninge, Next-generation thermo-plasmonic technologies and plasmonic nanoparticles in optoelectronics, Prog. Quant. Electron. 41 (2015) 23-70.

[3] P.K. Sahoo, D. Wang, P. Schaaf, Tunable plasmon resonance of semi-spherical nanoporous gold nanoparticles, Mater. Res. Express 1 (2014), 35018-10.

[4] L. Guoa, J.A. Jackmanb, H.H. Yang, P. Chen, N.J. Choa, D.H. Kim, Strategies for enhancing the sensitivity of plasmonic nanosensors, Nano Today 10 (2015) 213-239.

[5] D. Wang, R. Ji, A. Albrecht, P. Schaaf, Ordered arrays of nanoporous gold nanoparticles, Belstein J. Nanotechnol. 3 (2012) 651-657.

[6] M. Loncaric, J.S. Parramon, H. Zorc, S. Segota, P. Dubcek, S. Bernstorff, Optical and structural characterization of gold island films on glass substrates, Thin Solid Films 591 (2015) 204-209.

[7] A. Vaskevich, I. Rubinstein, Localized surface plasmon resonance (LSPR) transducers based on random evaporated gold island films: properties and sensing applications, in: A. Dmitriev (Ed.), Nanoplasmonic Sensors, Springer, New York, 2012, pp. 333-368.

[8] J. Siegel, O. Kvitek, O. Lyutakov, A. Reznickova, V. Svorcik, Low pressure annealing of gold nanostructures, Vacuum 98 (2013) 100-105.

[9] R. Alvarez, J.M. García-Martín, M. Macías-Montero, L. Gonzalez-Garcia, J.C. González, V. Rico, J. Perlich, J. Cotrino, A.R. González-Elipe1, A. Palmero, Growth regimes of porous gold thin films deposited by magnetron sputtering at oblique incidence: from compact to columnar microstructures, Nanotechnology 24 (2013) 045604-045609.

[10] Xin Sun, Hao Li, The influence of cyclic deposition and anneal on growth of isolated and well-dispersed Au nanoisland arrays, Appl. Surf. Sci. 357 (2015) 1836-1842.

[11] C.F. Klingshirn, A. Waag, A. Hoffmann, J. Geurts, Zinc Oxide-From Fundamental Properties Towards Novel Applications, Springer, 2010.

[12] K.A. Willets, R.P. van Duyne, Localized surface plasmon resonance spectroscopy and sensing, Annu. Rev. Phys. Chem. 58 (2007) 267-297.

[13] P.K. Jain, W. Huang, M.A. El-Sayed, On the universal scaling behavior of the distance decay of plasmon coupling in metal nanoparticle pairs: a plasmon ruler equation, Nano Lett. 7 (7) (2007) 2080-2088.

[14] O. Szabo, S. Flickyngerova, T. Ignat, I. Novotny, V. Tvarozek, Gold nanostructures sputtered on zinc oxide thin film and corning glass substrates, Facta Univ. Electron. Energ. 29 (1) (2016) 77-88.

[15] C. Ratscha, J.A. Venables, Nucleation theory and the early stages of thin film growth, J. Vac. Sci. Technol. A 21 (5) (2003) 96-109,

[16] C.A. Schneider, W.S. Rasband, K.W. Eliceiri, NIH Image to ImageJ: 25 years of image analysis, Nat. Methods 9 (2012) 671-675.

[17] P.K. Jain, M.A. El-Sayed, Surface plasmon coupling and its universal size scaling in metal nanostructures of complex geometry: elongated particle pairs and nanosphere trimers, J. Phys. Chem. C 112 (2008) 4954-4960.

[18] M.A. Otte, B. Sepulveda, in: A. Dmitriev (Ed.), Figure of Merit for Refractometric LSPR Biosensing, in Nanoplasmonic Sensors, Springer, New York, 2012, pp. 317-331. 